\title{
ANÁlise dE DEMANDAS DE PACIENTES E PROFISSIONAIS DE SAÚDE QUANTO À POLTRONA UTILIZADA PARA A ASSISTÊNCIA DO PACIENTE
}

\author{
ANALYSIS OF THE DEMANDS OF PATIENTS AND HEALTHCARE \\ PROFESSIONALS REGARDING THE ARMCHAIR USED IN PATIENT CARE
}

\author{
Fabiane Espíndola Gomes ${ }^{1}$, Helena Barreto dos Santos², \\ Flávio Sanson Fogliatto ${ }^{3}$, Ricardo Bertoglio Cardoso ${ }^{3}$
}

\section{RESUMO}

Introdução: Sistemáticas transversais permitem identificar soluções tecnológicas que melhor atendam às necessidades dos usuários, sendo assim críticas para otimização de recursos financeiros e melhoria da qualidade do serviço prestado. Dessa forma, o presente projeto de pesquisa teve por objetivo realizar uma análise transversal das demandas dos usuários finais, profissionais da saúde e pacientes, quanto às poltronas utilizadas para assistência no Serviço de Onco-Hematologia do Hospital de Clínicas de Porto Alegre.

Métodos: Entrevistas semiestruturadas com perguntas abertas foram conduzidas no próprio local de atendimento em cada unidade de saúde: Ambulatório de Quimioterapia, Banco de Sangue e Hospital Dia. A população da pesquisa foi definida por meio da identificação dos usuários com forte interação com a poltrona para assistência e seu tamanho considerando o critério de ponto de saturação.

Resultados: Foram entrevistados 15 profissionais de saúde e 16 pacientes entre setembro de 2015 e janeiro de 2016. Verificou-se que o conforto foi a demanda mais indicada por profissionais de saúde e pacientes, sendo as demais demandas similares, independentemente do tempo de atendimento, número de pacientes por turno ou carga horária de trabalho.

Conclusões: Acredita-se que a análise transversal das demandas dos usuários possibilita a melhora da qualidade da assistência e otimização do emprego de recursos financeiros por permitir a seleção de soluções tecnológicas que melhor atendem às necessidades dos usuários finais, tais como pacientes e profissionais de saúde.

Palavras-chave: Profissional da saúde; tomada de decisões; ergonomia

\section{ABSTRACT}

Introduction: The cross-sectional analysis allows managers to identify technological solutions that best meet users' needs, which are critical for optimizing costs and improving service quality. Therefore, this paper aimed at applying a cross-sectional analysis to identify the demands of end users, healthcare professionals, and patients, related to the armchairs available at the Onco-Hematology department of the Hospital de Clínicas de Porto Alegre.

Methods: Semi-structured interviews were conducted using open questions at each healthcare facility: Chemotherapy Clinic, Blood Bank, and Day Hospital. The study group was selected by identifying users with strong interaction with the armchair and the population sample size was defined by the saturation point criterion.

Results: A total of 15 health professionals and 16 patients were interviewed between September 2015 and January 2016. It was observed that comfort was the most
Clin Biomed Res. 2017;37(3):187-192

1 Programa de Residência Integrada Multiprofissional em Saúde (RIMS), Hospital de Clínicas de Porto Alegre (HCPA). Porto Alegre, RS, Brasil.

2 Assessoria de Operações Assistenciais, Hospital de Clínicas de Porto Alegre (HCPA). Porto Alegre, RS, Brasil.

3 Programa de Pós-graduação em Engenharia de Produção, Universidade Federal do Rio Grande do Sul (UFRGS). Porto Alegre, RS, Brasil.

Autor correspondente: Fabiane Espíndola Gomes fabiegomes@gmail.com Hospital de Clínicas de Porto Alegre (HCPA)

Rua Ramiro Barcelos, 2350, Bonfim 90035-903, Porto Alegre, RS, Brasil. 
indicated demand both by healthcare professionals and patients, and the remaining demands were similar, regardless of duration of care, number of patients per shift, and working hours.

Conclusions: A cross-sectional analysis of users' demands improves the quality of care provided and cost optimization rates, as it enables the selection of technological solutions better suited to the needs of end users, such as patients and healthcare professionals.

Keywords: Healthcare professionals; decision making; human engineering

A aquisição de equipamentos médicos representa um percentual importante do orçamento anual de hospitais, tornando a busca pela maximização do custo-benefício uma prioridade para gestores. Entretanto, a perspectiva do gestor não deve ser a única a ser considerada. É necessário estabelecer um processo de tomada de decisão que sistematicamente analise, estruture e forneça acesso a evidencias relevantes à decisão, diminuindo o risco de se negligenciar ou ignorar uma informação importante quanto ao uso ou aplicação do equipamento em análise ${ }^{1}$.

Um produto adequadamente projetado deve ser seguro, eficiente, durável, economicamente atraente e agradável ao uso. Tais atributos adicionam valor ao produto e todos eles, com exceção do preço, envolvem aspectos ergonômicos diretamente percebidos por usuários. A adição de valor a produtos requer uma abordagem sistemática de projeto, que considere as necessidades e capacidades dos usuários ${ }^{2}$.

Dessa forma, a implantação de sistemáticas transversais para identificar soluções tecnológicas que melhor atendam às necessidades dos diferentes usuários finais, e não apenas da direção médica ou da chefia de serviço, se faz fundamental em um contexto hospitalar ${ }^{3}$. Entretanto, essas sistemáticas, já consagradas em outros contextos, como na gestão de processos industriais, ainda carecem de amadurecimento dentro da gestão hospitalar, sendo necessário adaptá-las à realidade do atendimento em saúde 4 .

A Análise de Decisão com Critérios Múltiplos (MCDA - Multiple Criteria Decision Analysis), por exemplo, designa de forma coletiva uma série de técnicas que são úteis para tratar da variabilidade dos fatores que são considerados, assim como dos diferentes valores e critérios que são utilizados em tomadas de decisões que envolvam múltiplos atores ${ }^{5}$.

Entre as diferentes técnicas de MCDA, destacam-se o Processo Analítico Hierárquico (AHP - Analytic Hierarchy Process), uma ferramenta de apoio à tomada de decisões que pode ser utilizada na solução de problemas complexos ${ }^{6,7}$, e o Desdobramento da Função Qualidade (QFD - Quality Function Deployment), que permite a melhoria da qualidade do design de produtos através da priorização de suas características essenciais.
O projeto de ampliação do Hospital de Clínicas de Porto Alegre (HCPA), primeiro realizado desde sua fundação há 40 anos, possibilitará uma importante modernização de diferentes serviços do hospital. A previsão de aquisição de materiais hospitalares que possam beneficiar a qualidade da assistência aos usuários apresentou, assim, a necessidade da implantação de sistemáticas transversais, adaptadas da gestão de processos industriais, para análise de demandas.

Nesse contexto, a enfermagem está diretamente envolvida no processo de cuidado e se torna uma ferramenta importante no momento da escolha de novas tecnologias e mobiliários para melhor atender seus pacientes, visando à segurança e conforto destes. Dentre os equipamentos mais utilizados pelas equipes de enfermagem e pacientes, pode-se ressaltar a poltrona hospitalar, presente em diferentes formatos nos mais variados setores.

A ergonomia, área crítica para caracterização de um mobiliário, estuda a relação entre o ser humano e seu ambiente de trabalho, oferecendo aos profissionais conforto adequado, prevenção de acidentes e de doenças específicas. $\mathrm{O}$ ambiente de trabalho deve ser composto com itens que atendam às necessidades de cada indivíduo, oferecendo a eles as condições fundamentais para seu bem estar, acarretando adaptações que vão além de cadeiras ergonômicas, abrangendo todos os objetos que ligam o homem ao seu trabalho. Entre os benefícios da ergonomia incluem-se a redução de doenças ocupacionais, do cansaço excessivo e do desconforto no ambiente de trabalho. Adicionalmente, é comprovado que funcionários que trabalham em um local adequado têm um aumento de produtividade e da rentabilidade do trabalho ${ }^{8}$.

Somado à opinião da enfermagem, a inclusão do paciente se faz fundamental no processo de melhoria dos serviços de saúde. A Organização Mundial da Saúde (OMS), em 2006, desenvolveu um manual sobre a segurança do paciente e o programa "Pacientes pela Segurança do Paciente em Serviços de Saúde", o qual incentiva o envolvimento do paciente e de seus familiares no cuidado, colocando a voz do paciente no centro da questão. A participação do paciente no seu cuidado melhora a eficiência 
nos serviços de saúde, reduz as chances de erros e aumenta a segurança ${ }^{9}$.

Dessa maneira, como passo inicial na implantação de um processo transversal para seleção de mobiliário no HCPA, o presente projeto de pesquisa teve por objetivo realizar uma análise transversal das demandas de usuários finais, profissionais da saúde e pacientes, quanto às poltronas utilizadas para assistência no Serviço de Onco-Hematologia do HCPA.

\section{MÉTODOS}

O presente estudo, caracterizado como qualitativo, foi desenvolvido no HCPA. Como primeira atividade, a equipe do projeto reuniu-se com a finalidade de definir qual seria o mobiliário objeto da avaliação. Foi escolhido como objeto do estudo a poltrona para atendimento de pacientes, pois verificou-se que vários setores do hospital realizam seus atendimentos aos pacientes sentados em poltronas.

Entrevistas semiestruturadas com perguntas abertas foram conduzidas no próprio local de atendimento, permitindo ao participante responder livremente. A entrevista foi realizada por dois pesquisadores do projeto, devidamente treinados no Ambulatório de Quimioterapia, Banco de Sangue e Hospital Dia, os quais fazem parte do Serviço de Onco-Hematologia do HCPA. A população da pesquisa foi definida por meio da identificação dos usuários (profissionais de saúde e pacientes) com forte interação com a poltrona para assistência e o tamanho da amostra foi definido considerando o critério de ponto de saturação, conforme apresentado por Thiry-Cherques ${ }^{10}$.

Foram utilizados os seguintes critérios de inclusão para os profissionais de saúde: ser enfermeiro ou técnico de enfermagem e trabalhar no serviço onde foi identificada a utilização da poltrona; não foi adotado nenhum critério de exclusão. Como critérios de inclusão dos pacientes foram adotados: estar em tratamento no serviço onde foi identificada a utilização da poltrona e ser maior de 18 anos; os critérios de exclusão foram: ausência de condições clínicas para realizar a entrevista (sonolento, confuso, cansado), conforme orientação do profissional de saúde. Os participantes foram apresentados ao projeto e convidados a participar da entrevista com duração em torno de 15 a 30 minutos, respeitando a disponibilidade e condições dos pacientes e profissionais. Todos os participantes concordaram e assinaram o Termo de Consentimento Livre e Esclarecido (TCLE) em duas vias, uma ficando com o participante e outra com o pesquisador. Os dados coletados foram registrados no programa Excel $^{\circledR} 2013$, para posterior análise e interpretação das demandas.

Este estudo foi aprovado pelo Comitê de Ética em Pesquisa do HCPA (CEP/HCPA, projeto sob $\left.n^{\circ}: 15-0315\right)$ e conduzido em conformidade com a Resolução 466/12 do Conselho Nacional de Saúde.

\section{RESULTADOS}

Foram entrevistados 15 profissionais de saúde e 16 pacientes (Tabela 1 ), nas unidades selecionadas, entre setembro de 2015 e janeiro de 2016. A entrevista abrangeu perguntas sobre o tempo de uso da poltrona pelo usuário, padrão de uso da poltrona, a impressão do usuário sobre a poltrona e o que ele consideraria uma melhora necessária.

Os profissionais de saúde entrevistados apresentaram carga horária equivalente (Tabela 2 ), pois o turno de trabalho é de 6 horas diárias (a complementação da carga horária ocorre conforme organização de cada setor). Os profissionais do Hospital Dia entrevistados atenderam uma média de 18,3 pacientes/turno, apresentando uma maior rotatividade/demanda de pacientes se comparado ao Banco de Sangue, que atende em torno de 9,7 pacientes/turno (Tabela 2).

As demandas trazidas pelos profissionais de saúde dos setores entrevistados (Gráfico 1) foram semelhantes e referem-se à melhoria da qualidade da poltrona no momento assistencial, sendo elas: apresentar mobilidade/posicionamento do paciente (para atendimento às emergências/PCR), possuir apoio móvel para os braços, controle de altura (controle eletrônico), suporte para materiais e soro/ medicação, ser de material leve e de fácil higienização, apresentar tamanhos especiais (para pacientes obesos), apresentar travas de segurança nas rodas, bem como proporcionar conforto ao paciente (assento macio) e ao atendimento do mesmo.

Tabela 1: Número de profissionais de saúde e pacientes do HCPA entrevistados por setor participante da pesquisa.

\begin{tabular}{|c|c|c|c|}
\hline \multicolumn{2}{|c|}{$\begin{array}{c}\text { Distribuição dos usuários } \\
\text { entrevistados }\end{array}$} & \multirow{2}{*}{$\frac{\mathbf{N}^{\circ}}{5}$} & \multirow[t]{2}{*}{ Total } \\
\hline \multirow{3}{*}{ Enfermeiros } & $\begin{array}{l}\text { Ambulatório de } \\
\text { Quimioterapia }\end{array}$ & & \\
\hline & $\begin{array}{l}\text { Banco de } \\
\text { sangue }\end{array}$ & 3 & 9 \\
\hline & Hospital Dia & 1 & \\
\hline \multirow{3}{*}{$\begin{array}{l}\text { Técnicos de } \\
\text { enfermagem }\end{array}$} & $\begin{array}{l}\text { Ambulatório de } \\
\text { Quimioterapia }\end{array}$ & - & \\
\hline & $\begin{array}{l}\text { Banco de } \\
\text { sangue }\end{array}$ & 4 & 6 \\
\hline & Hospital Dia & 2 & \\
\hline \multirow{3}{*}{ Pacientes } & $\begin{array}{l}\text { Ambulatório de } \\
\text { Quimioterapia }\end{array}$ & 5 & \\
\hline & $\begin{array}{l}\text { Banco de } \\
\text { sangue }\end{array}$ & 8 & 16 \\
\hline & Hospital Dia & 3 & \\
\hline
\end{tabular}

Fonte: Elaborado pelo Autor. 
Observou-se que os pacientes atendidos no Ambulatório de Quimioterapia permanecem sentados nas poltronas em média 4,6 horas/dia, o dobro de tempo dos atendidos no Banco de Sangue (Tabela 2). Para os pacientes que permanecem por longos e médios períodos utilizando as poltronas, a principal demanda diz respeito ao conforto, especialmente do assento e encostos (Gráfico 2). O conforto implica na poltrona ser mais macia (material), possuir ajustes de posições independentes (costas e pernas), encosto para cabeça e braços, suporte para auxílio de alimentação, controle eletrônico para movimentação das posições (sentado/deitado), e tamanhos especiais (pacientes obesos).

Tabela 2: Descrição do tempo médio de utilização da poltrona pelos usuários para receber ou prover atendimento em cada setor participante da pesquisa.

\begin{tabular}{cccc}
\hline Local & Entrevistado & $\begin{array}{c}\text { Média de tempo de utilização } \\
\text { da poltrona (horas) }\end{array}$ & $\begin{array}{c}\text { Média de paciente atendido/profissional } \\
\text { (turno de } \mathbf{6} \text { horas) }\end{array}$ \\
\hline Ambulatório de & Profissional & 6,0 & 11,8 \\
\cline { 2 - 4 } Quimioterapia & Paciente & 4,6 & -- \\
\hline \multirow{2}{*}{ Banco de Sangue } & Profissional & 6,0 & 9,7 \\
\cline { 2 - 4 } & Paciente & 2,2 & -- \\
\hline \multirow{2}{*}{ Hospital Dia } & Profissional & 6,0 & 18,3 \\
\cline { 2 - 4 } & Paciente & 3,7 & -- \\
\hline
\end{tabular}

Nota: Todos os profissionais de saúde que participaram das entrevistas cumprem turnos de 6 horas nos setores onde trabalham, sendo essa quantidade de horas considerada como utilização do mobiliário para fornecer atendimento à pacientes. Fonte: Elaborado pelo Autor.

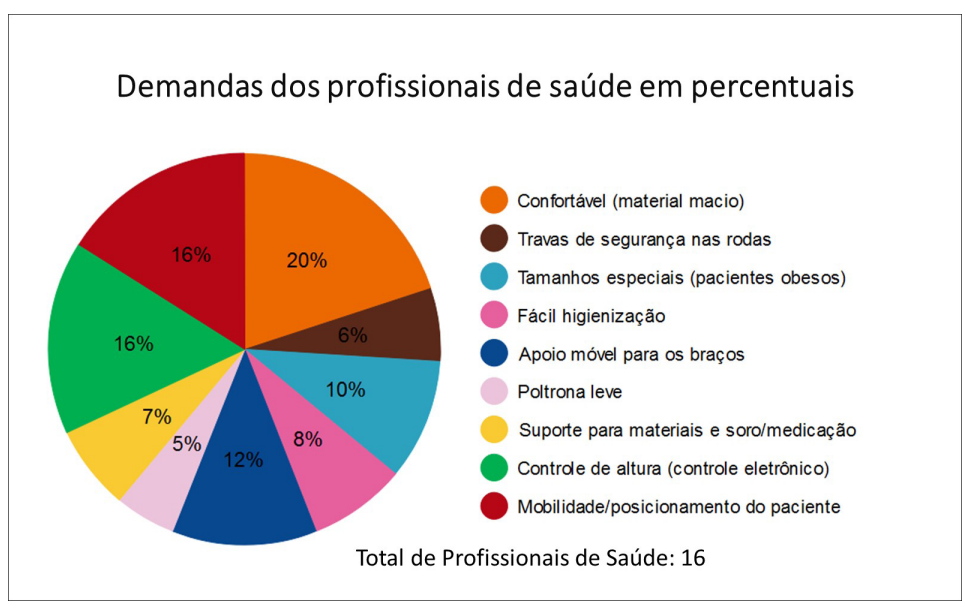

Gráfico 1: Demandas dos profissionais de saúde, em percentuais, coletadas nas entrevistas semiestruturadas. Fonte: Elaborado pelo Autor.

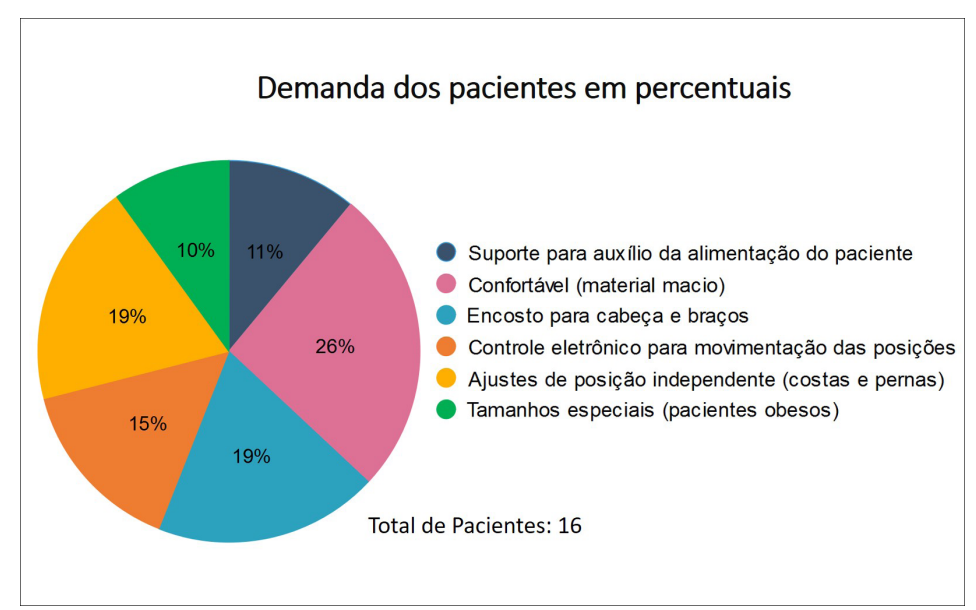

Gráfico 2: Demandas dos pacientes, em percentuais, coletadas nas entrevistas semiestruturadas. Fonte: Elaborado pelo Autor. 
Durante as entrevistas, alguns participantes apresentaram dúvidas sobre a pesquisa, mesmo após serem apresentados ao projeto, por nunca terem participado de processo semelhante dentro do hospital. Os profissionais relataram que se sentiram empoderados pelo processo, pois são usuários das poltronas e observam as necessidades reais do trabalho diário.

\section{DISCUSSÃO}

Este estudo analisou as demandas de usuários finais, profissionais da saúde e pacientes que fazem uso de poltronas nas seguintes unidades: Ambulatório de Quimioterapia, Banco de Sangue e Hospital Dia do HCPA.

Dentre os aspectos positivos da pesquisa, destaca-se a proximidade do pesquisador com o local, objeto e sujeito no momento da entrevista. Por ser uma entrevista semiestruturada com perguntas abertas, favoreceu-se um diálogo livre e espontâneo entre entrevistado e entrevistador. A utilização dessa modalidade de entrevista buscou facilitar a análise, compreensão e avaliação do problema de decisão.

Dentre os aspectos limitantes, pode-se destacar a dificuldade da realização das entrevistas devido às condições clínicas do paciente (sinalizada pela enfermeira responsável) e à sua pouca disponibilidade, assim demandando do pesquisador um maior número de visitas a cada setor.

A complexidade dos cuidados em saúde e dos pacientes, bem como as relações de trabalho dentro de um hospital, nem sempre permitem a participação do paciente e do profissional colaborador em decisões importantes que dizem respeito ao seu processo de cuidado (paciente) ou de trabalho (profissional de saúde).

O papel do enfermeiro transpõe a assistência direta ao paciente, visto que este assume funções gerenciais que permitem uma participação efetiva nas tomadas de decisões. A participação do enfermeiro no processo de seleção de mobiliário não é recorrente nos hospitais de grande porte, pois esses possuem setores especializados que realizam esta tarefa específica. Dessa maneira, muitos profissionais de saúde desconhecem o modelo de seleção e de compra dos materiais hospitalares ${ }^{11,12}$. Na presente pesquisa, esse desconhecimento (e o desejo de aprender) pôde ser verificado no momento da entrevista, tendo em vista as dúvidas apresentadas pelos profissionais sobre o processo, bem como sua intenção de participar de outras pesquisas que envolvam a melhoria na assistência.

O profissional de saúde, como uma de suas competências, realiza o processo de educação em saúde do paciente, desenvolvendo o conhecimento, as habilidades e o autoconhecimento necessários para assumir efetivamente a responsabilidade com as decisões acerca da sua saúde/doença/tratamento.
A participação do paciente na decisão acerca do seu cuidado vem sendo estimulada dentro dos grandes centros de saúde ${ }^{13}$. Ele pode desempenhar os papéis de decisor, comprador e usuário dos serviços de saúde; caso não tenha condições de decisão, outro membro da família pode desempenhar este papel. Esse envolvimento do paciente favorece o aumento da autonomia e responsabilidade do mesmo no seu cuidado e promove o fortalecimento da confiança na equipe assistencial, contribuindo assim para a redução das taxas de incidentes e erros ${ }^{14}$.

A ergonomia visa a melhorar as condições de trabalho dos profissionais, principalmente em relação aos equipamentos utilizados cotidianamente, tendo o potencial de prevenir diferentes acidentes no local de trabalho $0^{8,15}$. A enfermagem está diariamente exposta aos fatores de riscos ergonômicos, os quais ocasionalmente provocam desgastes físicos e psicológicos ${ }^{16}$. Nas entrevistas realizadas, tanto profissionais quanto pacientes relataram a importância do uso de poltronas funcionais que se adequem ao trabalho do profissional e, ao mesmo tempo, ao perfil dos pacientes que permanecem horas em tratamento.

Dentre as demandas relatadas pelos entrevistados, itens geralmente considerados relevantes como cinto de segurança, barreiras laterais e botões de alarme não se mostraram presentes ${ }^{17}$. Essa ausência, talvez incoerente em outros setores que não os estudados, ilustra as diferentes necessidades de cada setor e o potencial impacto negativo que a generalização do processo de seleção pode ter para a qualidade do serviço prestado.

No entanto, os resultados do presente estudo também sugerem que setores com pacientes e fluxo assistencial diferentes apresentam necessidades semelhantes às do atendimento onco-hematológico em relação às poltronas, independentemente do tempo de realização dos procedimentos, número de pacientes por turno e carga horária de trabalho.

A participação dos usuários finais no processo de tomada de decisão permite uma seleção de mobiliários mais alinhada às necessidades dos mesmos e garante uma análise mais próxima da realidade. Entretanto, fica clara a importância de se realizar um número maior de estudos que evidenciem esse processo, permitindo assim comparações mais extensivas de achados. Acredita-se que a análise transversal das demandas dos usuários possibilita a melhora da qualidade da assistência e a otimização do emprego de recursos financeiros por permitir a seleção de soluções tecnológicas que melhor atendam às necessidades dos usuários finais, tais como pacientes e profissionais de saúde.

\section{Conflito de interesse}

Os autores declaram não haver conflito de interesse. 


\section{REFERÊNCIAS}

1. Goetghebeur MM, Wagner M, Khoury $H$, Levitt RJ, Erickson LJ, Rindress D. Bridging health technology assessment (HTA) and efficient health care decision making with multicriteria decision analysis (MCDA): applying the EVIDEM framework to medicines appraisal. Med Decis Making. 2012;32:376-88.

2. Cushman W, Rosenberg D. Human factors in product design. Amsterdam: Elsevier Science; 1991.

3. Drummond MF, Schwartz JS, Jönsson B, Luce BR, Neumann PJ, Siebert U, et al. Key principles for the improved conduct of health technology assessments for resource allocation decisions. Int J Technol Assess Health Care. 2008;24:244-58.

4. Kuljis J, Paul RJ, Stergioulas LK, editores. Can health care benefit from modeling and simulation methods in the same way as business and manufacturing has? In: Proceedings of the Winter Simulation Conference; 2007 Dec 9-12; Washington. Washington; 2007. p. 1449-53.

5. Thokala P, Devlin N, Marsh K, Baltussen R, Boysen M, Kalo Z, et al. Multiple criteria decision analysis for health care decision making: an introduction. Report 1 of the ISPOR MCDA emerging good practices task force. Value Health. 2016;19:1-13.
6. Liberatore MJ, Nydick RL. The analytic hierarchy process in medical and health care decision making: a literature review. Eur J Oper Res. 2008;189:194-207.

7. Saaty TL. The analytic hierarchy process: planning, priority setting, resource allocation. New York: McGraw-Hill; 1980.

8. Daher MJE, Oliveira MM, Vidal LL, Paes GK. A importância da utilização da ergonomia para a saúde do trabalhador. Rev Pesq. 2011;3:166266.

9. Brasil. Ministério da Saúde. Documento de referência para o Programa Nacional de Segurança do Paciente. Brasília: Ministério da Saúde, Fundação Oswaldo Cruz, ANVISA; 2014. [citado 2017 Fev 24]. Disponível em: http://bvsms.saude. gov.br/bvs/publicacoes/documento referencia_programa_nacional_ seguranca.pdf

10. Thiry-Cherques HR. Saturação em pesquisa qualitativa: estimativa empírica de dimensionamento. Rev PMKT. 2009;3:20-7.

11. Oliveira NC, Chaves LDP. Gerenciamento de recursos materiais: o papel da enfermeira de unidade de terapia intensiva. Rev Rene Fortaleza. 2009;10:19-27.
12. Siman A, Brito M, Carrasco M. Participação do enfermeiro gerente no processo de acreditação hospitalar. Rev Gaucha Enferm. 2014;35:93-9.

13. Campos DCF, Graveto JMGN. Papel do enfermeiro e envolvimento do cliente no processo de tomada de decisão clínica. Rev Lat Am Enfermagem. 2009;17:1065-70.

14. Silva TO, Bezerra ALQ, Paranaguá TTB, Teixeira CC. O envolvimento do paciente na segurança do cuidado: revisão integrativa. Rev Eletr Enf. 2016;18:e1173.

15. Kassada DS, Lopes FLP, Kassada DA, editores. Ergonomia: atividades que comprometem a saúde do trabalhador. In: Anais do VII Encontro Internacional de Produção Científica; 2011 Out 25-28; Foz do Iguaçu, Paraná. Maringá: CESUMAR; 2011.

16. Cavalcante CAA, Enders BC, Menezes RMP, Medeiros SM. Riscos ocupacionais do trabalho em enfermagem: uma análise contextual. Cienc Cuidado Saude. 2008;5:88-97.

17. Oliver D, Healey F, Haines TP. Preventing falls and fall-related injuries in hospitals. Clin Geriatr Med. 2010;26:645-92.

Recebido: Fev 24, 2017 Aceito: Jul 24, 2017 